\title{
Covid-19 and the need for perinatal mental health professionals: now more than ever before
}

\author{
Michael T. Hynan (ib)
}

Received: 16 April 2020 / Revised: 3 May 2020 / Accepted: 14 May 2020 / Published online: 27 May 2020

(c) Springer Nature America, Inc. 2020

When the healthcare system has endured the disaster of the Covid-19 pandemic, leaders of perinatal medicine will have many conversations. Topics will include re-analyses of priorities of care within hospitals, across healthcare systems and beyond. A greater role for perinatal mental health professionals must be part of these conversations.

Over the past years I have participated in actual and teleconference meetings of neonatal intensive care unit (NICU) psychologists across the United States [1]. Our conversations have included NICU parents and members of other perinatal disciplines: social workers, neonatologists, psychiatric nurses, etc. As of mid-March 2020, we have had weekly meetings for peer mentoring during the Covid crisis. Our participants are primarily NICU based but often extend to other hospital units.

Currently (early May 2020) most NICU social workers and psychologists are on the nonessential list, abilities of parents to engage with their babies vary widely and telemedicine services vary from nonexistent to competent (seldom reported). Most NICU psychologists find themselves on the early edges of the telemedicine learning curve.

The prevailing values and goals of any NICU have been replaced by the urgent need to curtail this pandemic. The national news contains updates on medical equipment shortages and construction of field hospitals. A top priority has been maintaining the health of care providers, with physical health dominating mental health concerns. But heroic descriptions of the psychological struggles of medical staff, victims, and survivors are beginning to emerge along with hospitals' attempts to buffer the emotional damage to staff.

Michael T. Hynan

hynan@uwm.edu

1 Department of Psychology, University of Wisconsin-Milwaukee, Milwaukee, WI, USA
In the coming months the death count related to the pandemic will help epidemiologists to estimate cases of depression, anxiety disorders, and posttraumatic stress (PTS) in the general population. Only then can we extrapolate from these numbers to guess at the psychological effects on workers in the perinatal field, parents, babies, and families. For now, the only question about the emotional outcomes can be, "How bad?".

For over 20 years research has indicated that, in ordinary times, the experience of a NICU hospitalization is a potentially traumatic event for the baby's parents [2-6]. A published estimate of the prevalence of diagnosable mental disorders in NICU parents in the first partum year is 20-30\% (or more) [5]. Additional NICU parents experience troublesome symptoms of depression, PTS, etc. that usually decline in intensity over the first year $[2,6]$. The highest report of parental PTS symptoms that I have seen in the literature $(60 \%$ of mothers and $47 \%$ of fathers exceeding threshold) comes from a NICU that had strict limitations on skin-to-skin care and breastfeeding (not allowed) and visitation curtailment to one parent at a time [3]. The current pandemic limitations on parental engagement with their NICU baby, and partners excluded from the birth of their baby, can only made a family's future worse.

Published in ordinary times, a survey of burnout rates in NICU staff in multiple hospitals in California indicated that $26.7 \%$ of respondents qualified for burnout by responding above threshold in endorsing items of emotional exhaustion [7]. The range across NICUs was 7.5-42.9\%. There is near universal acknowledgement that these rates are going to increase.

The predominant topic of the April 2020 NICU psychologists' discussions was strategies to support NICU staff. Members described their own support activities and reported hurried efforts of different hospitals to have psychiatry, social work, and psychology staff establish resources such as warm lines, signups for counseling sessions, debriefings, and meditation times. Despite all these efforts, it is likely that hospitals will lose more personnel to 
burnout than ever before. How to make use of what is learned now in planning perinatal care for the future?

Adding mental health staff in the perinatal setting often involves economics (even if most everyone agrees that it is the right thing to do). Our group has often heard from hospital administrators that the NICU cannot afford to add more social workers or a psychologist. What is a hospital's cost for training new nurses, doctors, and other staff? Likely the local costs for replacements exceed what it would take to add mental health professionals. Additional hiring could also be framed as the hospital's commitment to the broader well-being of both patients and staff. Elevating a hospital's morale in these times (and preparing for future stability in programming support structures for staff) should be a top priority. Perinatal mental health professionals must be included in these discussions.

Acknowledgements I appreciate the editorial assistance of Lauren Leslie-Hynan. These comments are dedicated to Cheryl Milford, one of the first NICU psychologists.

\section{Compliance with ethical standards}

Conflict of interest The author declares no conflict of interest.
Publisher's note Springer Nature remains neutral with regard to jurisdictional claims in published maps and institutional affiliations.

\section{References}

1. National Network of Neonatal Psychologists. www.nationalperina tal.org/psychologists. Accessed 15 Apr 2020.

2. Holditch-Davis D, Miles MS, Weaver MA, Black B, Beeber L, Thoyre S, et al. Patterns of distress in African-American mothers of preterm infants. J Dev Behav Pediatr. 2009;30:193-205.

3. Aftyka A, Rybojad B, Rosa W, Wrobel A, Karakula-Juchnowicz H. Risk factors for the development of posttraumatic stress disorder and coping strategies in mothers and fathers following infant hospitalized in the neonatal intensive care unit. J Clin Nurs. 2017;26: 338-47.

4. Hynan M, Steinberg Z, Baker L, Cicco R, Geller P, Lassen S, et al. Recommendations for mental health professionals in the NICU. J Perinatol. 2015;35:S14-8.

5. Hynan MT, Mounts KO, Vanderbilt DL. Screening parents of highrisk infants for emotional distress: rationale and recommendations. J Perinatol. 2013;33:748-53.

6. Bonanno GA, Westphal M, Anthony D, Mancini AD. Resilience to loss and potential trauma. Ann Rev Clin Psychol. 2011;7:511-35.

7. Tawfik DS, Phibbs CS, Sexton JB, Tan P, Sharek PJ, Nisbet CC, et al. Factors associated with provider burnout in the NICU. Pediatrics. 2017;139. https://doi.org/10.1542/peds.2016-4134. 